\title{
Multi-omics analyses of red blood cell reveal antioxidation mechanisms associated with hemolytic toxicity of gossypol
}

\author{
Chaohua Tang ${ }^{1,2}$, Qingshi Meng ${ }^{1,2}$, Kai Zhang ${ }^{1,2}$, Tengfei Zhan ${ }^{1,2}$, Qingyu Zhao ${ }^{1,2}$, \\ Sheng Zhang ${ }^{3}$ and Junmin Zhang ${ }^{1,2}$ \\ ${ }^{1}$ State Key Laboratory of Animal Nutrition, Institute of Animal Science, Chinese Academy of Agricultural Sciences, Beijing, \\ China \\ ${ }^{2}$ Scientific Observing and Experiment Station of Animal Genetic Resources and Nutrition in North China, Ministry of \\ Agriculture, Beijing, China \\ ${ }^{3}$ Institute of Biotechnology, Cornell University, Ithaca, NY, USA \\ Correspondence to: Junmin Zhang, email: zhjmxms@sina.com \\ Keywords: gossypol, red blood cell, hemolytic toxicity, metabolomics, proteomics \\ Received: July 21,2017 Accepted: September 21, 2017 Published: October 10, 2017 \\ Copyright: Tang et al. This is an open-access article distributed under the terms of the Creative Commons Attribution License 3.0 \\ (CC BY 3.0), which permits unrestricted use, distribution, and reproduction in any medium, provided the original author and source \\ are credited.
}

\section{ABSTRACT}

Gossypol is an antiproliferative drug with limited use due to its hemolytic toxicity. In this study, accelerated hemolysis was observed in the cows treated with gossypol. Comparative metabolomics were used to gain responsive pathways in the red blood cell (RBC) to the treatment, which were crossly validated by parallel iTRAQ-based proteomic analysis and enzyme activity assay. We found that gossypol treatment appeared to considerably activate pentose phosphate pathway (PPP) with an increased key product of ribose-5-phosphate and the increased abundance and activity of several key enzymes such as 6-phosphogluconate dehydrogenase, flavin reductase, and ribose-phosphate pyrophesphokinase. Meanwhile, a decreased glycolysis metabolism was observed, as many input metabolites of glycolysis were reduced in the gossypol group, whereas its distal metabolites were unchanged, along with decreased abundance of triosephosphate isomerase and increased abundance of enzymes catalyzing several distal glycolytic steps. Oxidative reduction pathways were also remarkably affected as we found a decreased substrate of flavin reductase, glutathione disulfide, increased glutathione reductase activity, and increased abundance and activity of glutathione S-transferase with the increase of its catalytic product, cysteine. Our results demonstrated that glycolysis, PPP, and oxidative reduction pathways of RBC were all involved in RBC's response to the hemolytic toxicity of gossypol.

\section{INTRODUCTION}

Gossypol is a polyphenolic compound derived from the cotton plant and has gained great interest due to its contraceptive activity. Besides its antifertility effects, other biological properties have also been reported [1, 2], of which antiproliferative effects have been recently drawn considerable attention $[3,4]$. The use of gossypol as an alternative anticancer drug is limited by two main side effects, which include (a) the occurrence of hypokalaemic paralysis [5] and (b) the acceleration of eryptosis, which may trigger hemolysis and anemia [6]. The available evidence on animals and man suggests that the direct toxic effect of gossypol on the renal tubules leads to the renal leakage of potassium and results in hypokalaemic paralysis [5]. However, the mechanism of the hemolytic toxicity of gossypol in vivo has not been reported.

The most important task of red blood cell (RBC) is to bind and transport oxygen, which requires passage through microcapillaries. The latter is achieved by a 
drastic modification of its biconcave shape, made possible only by the loss of the nucleus and cytoplasmic organelles and, consequently, the lack of ability to synthesize proteins [7]. Yet, a number of vital pathways are still active in $\mathrm{RBC}$, which contributed to generate energy and reducing power to perform its essential functions [8]. As soon as the RBC cannot fulfill its vital functions anymore, it will eventually lose its biconcave shape and eryptosis happens. Therefore, it is important to consider that RBC can accumulate modifications on metabolites and proteins that represent the long-term status of the body. However, the degree of hemolysis not only depends on the severity of RBC functions impairment, but also relies on the ability to compensate for the abnormalities by reticulocytes.

As a clinical sign of gossypol toxicity, the increases of RBC osmotic fragility induced by gossypol were reported extensively in animals $[9,10]$. In recent years, an in vitro study showed that treatment of $\mathrm{RBC}$ with gossypol stimulates $\mathrm{Ca}^{2+}$ entry and induces eryptosis [6]. The subsequent increase in cytosolic $\mathrm{Ca}^{2+}$ leads to activation of $\mathrm{Ca}^{2+}$-sensitive $\mathrm{K}^{+}$channels [11], resulting in $\mathrm{K}^{+}$exit, hyperpolarization, and $\mathrm{Cl}^{-}$exit $[12,13]$. Cellular $\mathrm{KCl}$ loss, together with osmotically obliged water, results in cell-membrane scrambling and cell shrinkage [6], hallmarks of suicidal erythrocyte death. Despite cationhomeostasis dysregulation, other mechanisms might also contribute to the hemolytic toxicity of gossypol. These include (a) gossypol binds to iron [14] and, in so doing, produce a gossypol-iron complex that may cause iron deficiency, thereby affecting hemoglobin (HGB) generation and influencing RBC function; (b) gossypol possesses antioxidant and pro-oxidant properties [15] and could induce oxygen-radical formation [16], an indicator of oxidative stress, which could trigger eryptosis [17]. Nevertheless, besides its cation-homeostasis dysregulation found in vitro [6], little is known about the gossypol effects in vivo. In this context, metabolomics and proteomics are the methods of choice to investigate the RBC's response to the hemolytic toxicity of gossypol at the molecular level, given the fact that the mature RBCs do not contain RNA or DNA [18-21].

In this study, an increased hemolysis was observed after gossypol treatment of dairy cows. Metabolomics study showed that glycolysis, pentose phosphate pathway, and oxidative reduction pathways were profoundly affected. A parallel proteomics analysis, combined with targeted-based enzyme assay, found that the abundance and activity change of most enzymes involved in the above pathways were in good agreement with the alteration of metabolome. Here, we reported multi-omics analysis results, which provided not only new insights into the molecular mechanisms on the $\mathrm{RBC}$ in response to the hemolytic toxicity of gossypol and but also shed light on the continuing studies of gossypol as a potential anticancer drug.

\section{RESULTS}

\section{Gossypol administration induces $\mathrm{RBC}$ hemolysis}

$\mathrm{RBC}$ parameters and percentage of hemolytic RBC in $0.8 \% \mathrm{NaCl}$-phosphate buffer are presented in Figure 1. $\mathrm{RBC}, \mathrm{HGB}$, and $\mathrm{MCHC}$ values between the control and gossypol-treated groups were not significantly different.
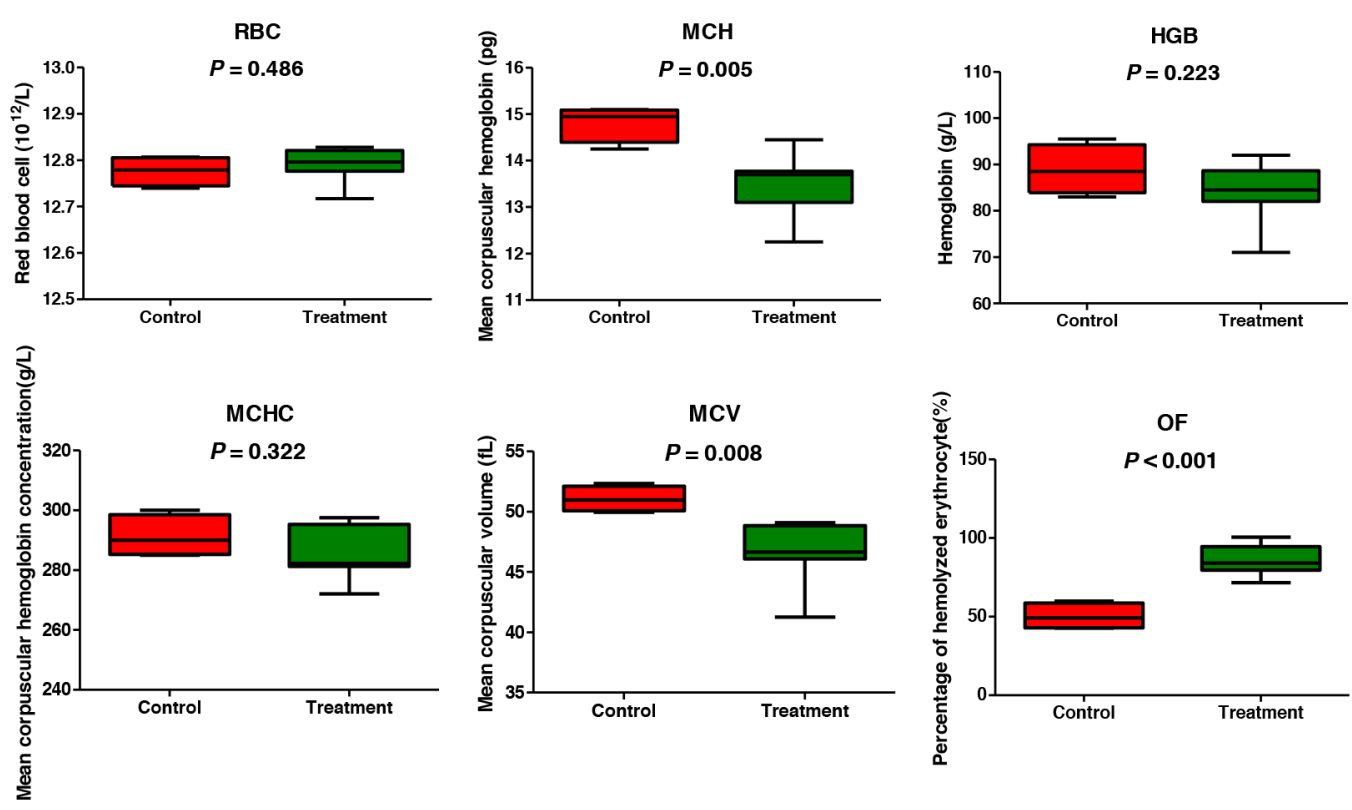

Figure 1: Red blood cell parameters and osmotic fragility of dairy cows from the control and gossypol-treated groups. 
$\mathrm{MCH}$ and MCV were significantly decreased by gossypol treatment ( $p=0.005$ and 0.008 , respectively), and the percentage of hemolytic RBC in $0.8 \%$ NaCl-phosphate buffer in the gossypol-treated group was $86.08 \%$, which was significantly higher than that observed in the control group $(50.28 \% ; p=0.00007)$.

\section{Quality control of metabolomics and proteomics analysis}

The data quality from GC-MS metabolomic analysis was evaluated according to the intesities of internal standards added during sample preparation. The relative standard derivations (RSDs) for the internal standards 13C3-15N-L-alanine, 13C5-15N-L-valine, 13C6-15N-Lleucine, and 13C6-15N-L-isoleucine were 5.08\%, 4.59\%,
$4.82 \%$, and $3.52 \%$, respectively. Additionally, the data quality from GC-MS and UPLC-QTOF metabolomics analysis was assessed by three quality control samples analyzed at the beginning, middle, and end of data acquisition. The RSDs for $91.78 \%$ and $72.54 \%$ features were $<30 \%$ in GC-MS and UPLC-QTOF metabolomic data sets, respectively. Proteomic data were assessed for the variation of identified and quantified proteins, which shown that the RSDs for $74.05 \%$ and $77.29 \%$ of the quantified proteins were $<30 \%$ in the control and gossypol-treated groups, respectively. Hierarchical cluster analysis of the differential metabolites and proteins displayed as heat maps clearly shows that samples were clustered into two groups (Figure 2). All these results suggested reproducible technical replicates and acceptable biological variation in this study.

(a)

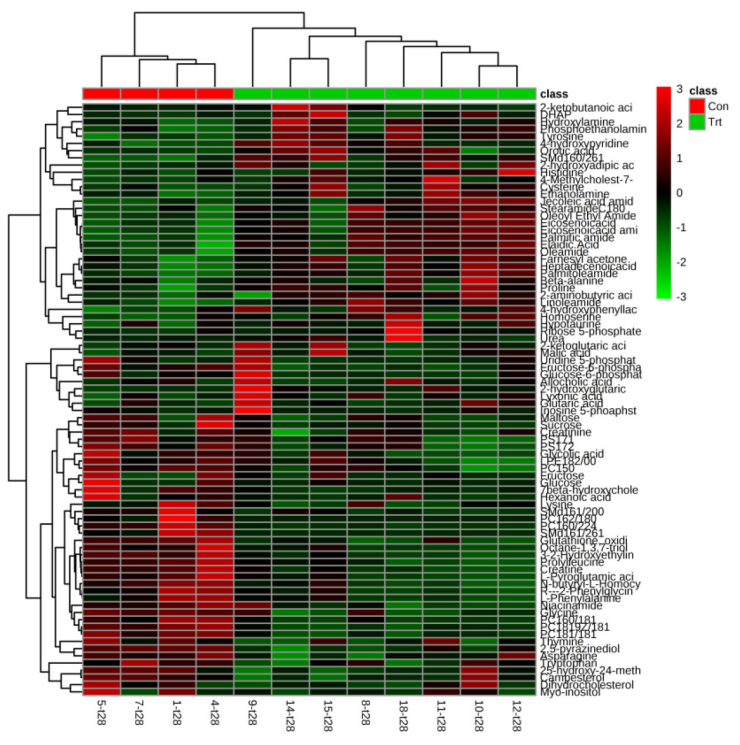

(b)

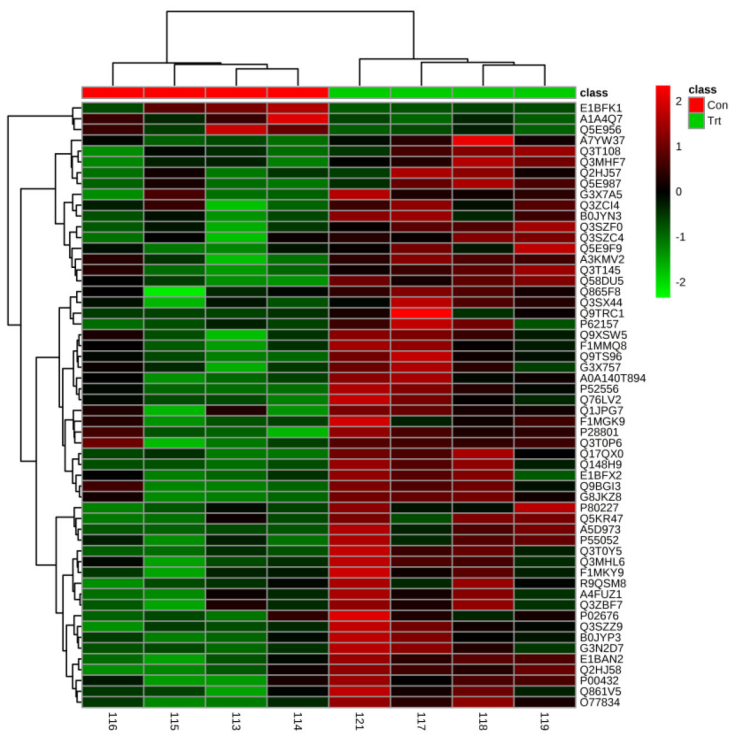

Figure 2: Heat map of all differential metabolites (a) and proteins (b) in red blood cells of cows from control and gossypol-treated groups. 


\section{Altered RBC metabolome by gossypol treatment}

Multivariate statistical analysis was used to detect metabolic differences in the GC-MS and UPLC-QTOF data sets between the control and gossypol groups (Figures 3). For GC-MS data, four principal components were calculated in PCA analysis, with an R2X value of 0.605 . There was a tendency of cluster between the control and gossypol-treated groups in the score plot of PCA, and the gossypol-treated group was well discriminated from the control group according to PLS-DA (Figure 3). Three principal components were calculated to build the PLSDA model with the following parameters: $\mathrm{R} 2 \mathrm{X}=0.414$, $\mathrm{R} 2 \mathrm{Y}=0.996$, and Q2 $=0.646$. The differential metabolites between the two groups were selected based on the $p$-values $(p<0.05)$ and the $\mathrm{FC}(\mathrm{FC}>1.4$ or $<0.67)$. A

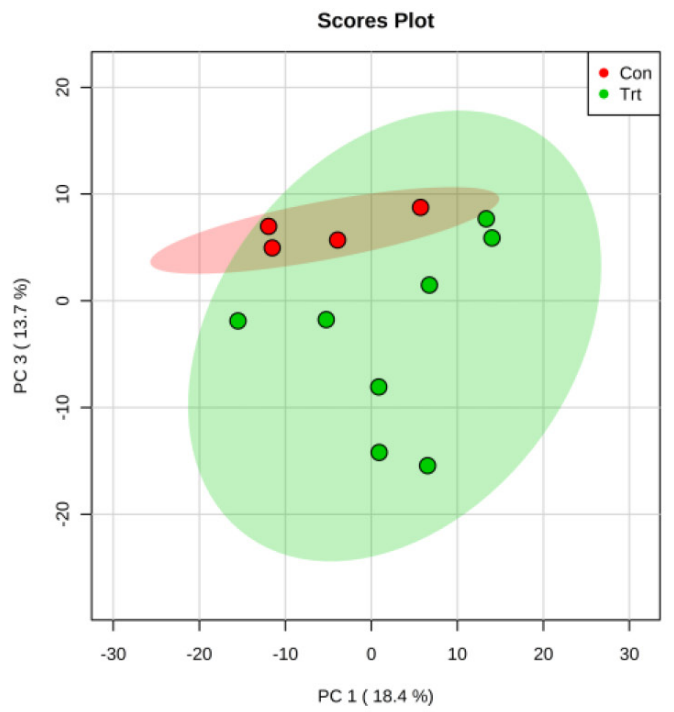

(a)

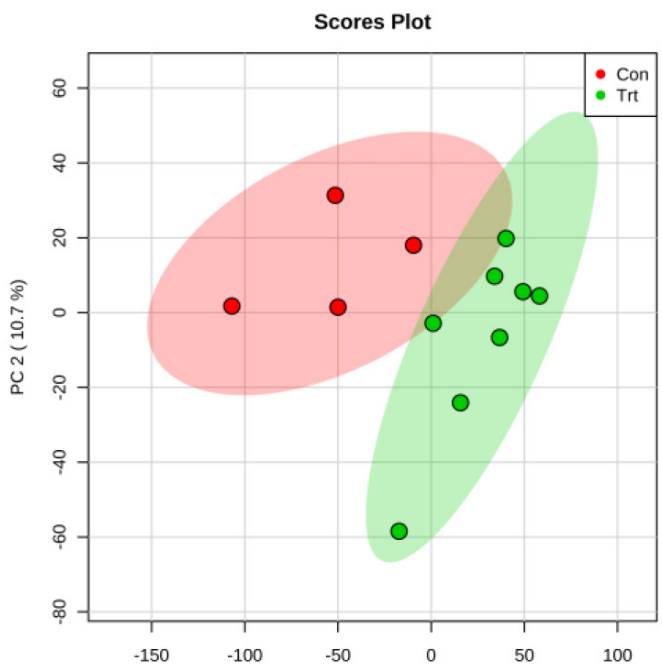

(c)

PC 1 ( $49.4 \%)$ total of 30 metabolites meeting any of the above criteria and being chosen for pathway analysis are shown in Table 1 and source dataset are presented in Supplementary Material 1.

The dataset derived from UPLC-QTOF analysis was examined similar to that described for the GC-MS dataset (Figure 3). Two principal components were calculated in the PCA, with $\mathrm{R} 2 \mathrm{X}=0.531$ and the control and gossypol groups clustered into two groups. The two groups were well discriminated according to the supervised methods PLS-DA. Three components were obtained in the PLS$\mathrm{DA}$, with $\mathrm{R} 2 \mathrm{X}=0.579, \mathrm{R} 2 \mathrm{Y}=0.999$, and $\mathrm{Q} 2=0.874$. Differential metabolites between the two groups were selected based on the criteria stated in the description of GC-MS data analysis. A total of 36 metabolites meet all the criteria were found, with the abundance of

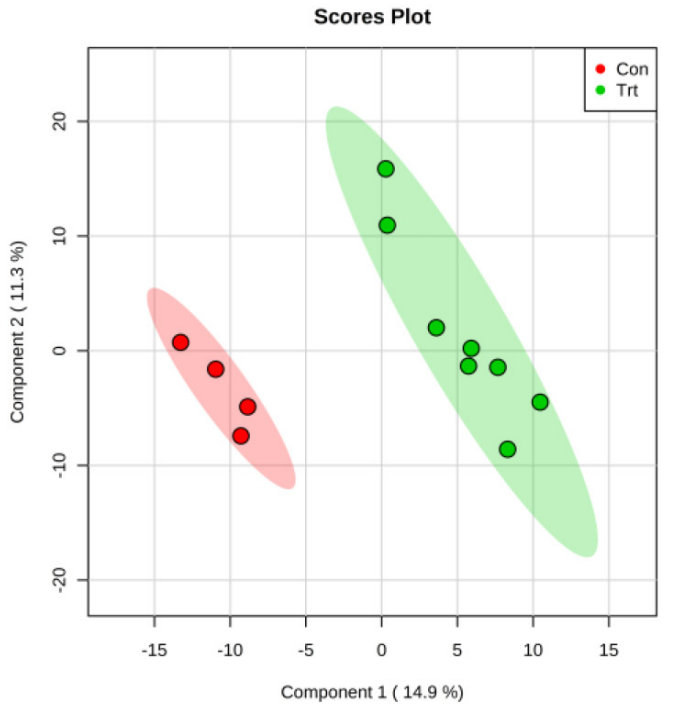

(b)

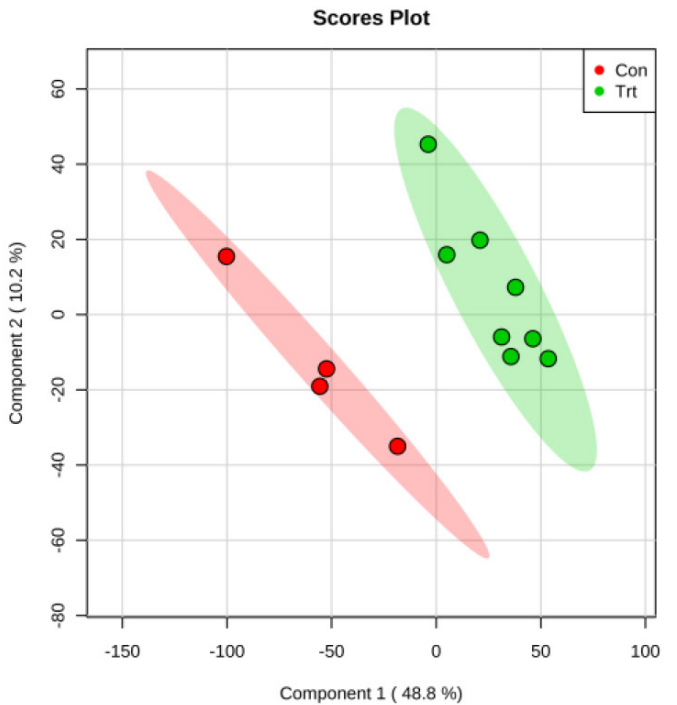

(d)

Figure 3: PCA and PLS-DA based on data derived from GC-MS (a, b) and UPLC-QTOF (c, d) metabolomics analysis of red blood cell from dairy cows from the control and gossypol-treated groups. 
Table 1: Differential metabolites screen for pathway analysis identified by GC-MS in red blood cells of cows from the control and gossypol-treated groups

\begin{tabular}{|c|c|c|c|c|c|}
\hline No. & $\mathbf{m} / \mathbf{z}$ & Rt (min) & $p$-value & FC & Compounds \\
\hline \multicolumn{6}{|c|}{$p$-value $<0.05$} \\
\hline 1 & 205 & 7.56 & 0.031 & 0.839 & Glycolic acid \\
\hline 2 & 102 & 8.5 & 0.004 & 0.568 & Glycine \\
\hline 3 & 152 & 9.17 & 0.017 & 1.153 & 4-hydroxypyridine \\
\hline 4 & 174 & 11.56 & 0.033 & 1.260 & Ethanolamine \\
\hline 5 & 241 & 13.58 & $<0.001$ & 0.571 & 2,5-pyrazinediol \\
\hline 6 & 243 & 16.56 & 0.034 & 0.808 & Asparagine \\
\hline 7 & 142 & 18.06 & 0.029 & 1.549 & Proline \\
\hline 8 & 188 & 21.02 & 0.038 & 1.484 & Phosphoethanolamine \\
\hline 9 & 179 & 22.28 & 0.006 & 1.397 & Tyrosine \\
\hline 10 & 179 & 22.41 & 0.047 & 1.711 & 4-hydroxyphenyllactic acid \\
\hline 11 & 311 & 23.78 & 0.015 & 0.508 & Palmitoleic acid \\
\hline 12 & 202 & 25.53 & 0.047 & 0.899 & Tryptophan \\
\hline 13 & 456 & 32.04 & 0.016 & 0.249 & 7beta-hydroxycholesterol \\
\hline 14 & 386 & 32.19 & 0.049 & 0.660 & 25-hydroxy-24-methylcholesterol \\
\hline 15 & 343 & 32.27 & 0.050 & 0.666 & Campesterol \\
\hline \multicolumn{6}{|c|}{ FC $>1.4$ or $<0.67$} \\
\hline 1 & 173 & 7.54 & 0.114 & 0.596 & Hexanoic acid \\
\hline 2 & 261 & 14.59 & 0.303 & 1.403 & Glutaric acid \\
\hline 3 & 218 & 15.5 & 0.086 & 1.449 & Homoserine \\
\hline 4 & 220 & 17.6 & 0.083 & 1.786 & Cysteine \\
\hline 5 & 129 & 18 & 0.158 & 3.940 & 2-hydroxyglutaric acid \\
\hline 6 & 198 & 18.01 & 0.425 & 1.712 & 2-ketoglutaric acid \\
\hline 7 & 129 & 19.53 & 0.137 & 1.453 & 2-hydroxyadipic acid \\
\hline 8 & 315 & 20.68 & 0.155 & 3.470 & Dihydroxy acetone phosphate \\
\hline 9 & 103 & 22.09 & 0.267 & 0.569 & Fructose \\
\hline 10 & 319 & 22.58 & 0.103 & 0.296 & Glucose \\
\hline 11 & 291 & 23.41 & 0.636 & 3.377 & Pantothenic acid \\
\hline 12 & 217 & 24.38 & 0.148 & 0.636 & Myo-inositol \\
\hline 13 & 315 & 24.53 & 0.321 & 1.699 & Ribose 5-phosphate \\
\hline 14 & 361 & 28.5 & 0.062 & 0.450 & Maltose \\
\hline 15 & 269 & 32.7 & 0.147 & 2.046 & 4-Methylcholest-7-en-3-ol \\
\hline
\end{tabular}

The $p$-value was calculated from Student's $t$ test. FC (fold change), defined as normalized peak area of metabolites in treatment group/control group. 
Table 2: Differential metabolites identified by UPLC-QTOF in red blood cells of cows from the control and gossypoltreated groups

\begin{tabular}{|c|c|c|c|c|c|}
\hline No. & Rt (min) & $\mathbf{m} / \mathbf{z}$ & $p$-value & FC & Compounds \\
\hline 1 & 15.84 & 619.6111 & 0.029 & 2.854 & Oleoyl Ethyl Amide \\
\hline 2 & 13.24 & 254.249 & 0.006 & 2.466 & Palmitoleamide \\
\hline 3 & 14.67 & 308.2958 & 0.031 & 1.916 & Jecoleic acid amide \\
\hline 4 & 15.49 & 284.2961 & 0.035 & 1.904 & Stearamide (C18:0) \\
\hline 5 & 13.57 & 263.2381 & 0.003 & 1.895 & Linoleamide \\
\hline 6 & 17.66 & 815.696 & 0.004 & 1.848 & SM (d16:0/26:1) \\
\hline 7 & 13.69 & 268.2636 & 0.009 & 1.821 & Heptadecenoic acid amide \\
\hline 8 & 15.55 & 310.3122 & 0.005 & 1.740 & Eicosenoic acid amide \\
\hline 9 & 13.41 & 263.2381 & 0.024 & 1.696 & Farnesyl acetone \\
\hline 10 & 14.06 & 256.2659 & 0.001 & 1.673 & Palmitic amide \\
\hline 11 & 15.55 & 293.2841 & 0.008 & 1.558 & Eicosenoic acid \\
\hline 12 & 14.41 & 282.2813 & 0.001 & 1.404 & Oleamide \\
\hline 13 & 14.41 & 265.2529 & 0.003 & 1.253 & Elaidic Acid \\
\hline 14 & 11.34 & 496.3402 & 0.019 & 0.673 & $\mathrm{PC}(15: 0)$ \\
\hline 15 & 10.79 & 478.2933 & 0.047 & 0.662 & $\operatorname{LPE}(18: 2 / 0: 0)$ \\
\hline 16 & 12.1 & 524.2974 & 0.050 & 0.662 & $\operatorname{PS}(17: 1)$ \\
\hline 17 & 0.96 & 114.0674 & 0.004 & 0.637 & Creatinine \\
\hline 18 & 0.79 & 130.0865 & 0.003 & 0.633 & L-Pyroglutamic acid \\
\hline 19 & 11.05 & 522.282 & 0.017 & 0.628 & $\operatorname{PS}(17: 2)$ \\
\hline 20 & 3.77 & 229.1559 & 0.001 & 0.617 & Prolylleucine \\
\hline 21 & 4.2 & 166.0874 & 0.011 & 0.606 & L-Phenylalanine \\
\hline 22 & 4.2 & 120.0819 & 0.008 & 0.588 & (R)-(-)-2-Phenylglycinol \\
\hline 23 & 1.02 & 132.0779 & 0.001 & 0.586 & Creatine \\
\hline 24 & 4.92 & 188.0722 & 0.001 & 0.586 & $\begin{array}{l}\text { N-butyryl-L-Homocysteine } \\
\text { thiolactone }\end{array}$ \\
\hline 25 & 6.67 & 163.134 & 0.004 & 0.503 & Octane-1,3,7-triol \\
\hline 26 & 1.78 & 123.0563 & 0.019 & 0.485 & Niacinamide \\
\hline 27 & 3.58 & 613.1584 & 0.001 & 0.433 & Glutathione, oxidized \\
\hline 28 & 14.16 & 144.0812 & $<0.001$ & 0.390 & 3-(2-Hydroxyethyl) indole \\
\hline 29 & 0.98 & 365.1049 & 0.042 & 0.357 & Sucrose \\
\hline 30 & 17.66 & 731.6012 & 0.022 & 0.231 & SM (d16:1/20:0) \\
\hline 31 & 17.65 & 786.5994 & $<0.001$ & 0.197 & $\operatorname{PC}(18: 1 / 18: 1)$ \\
\hline 32 & 14.25 & 786.5963 & $<0.001$ & 0.188 & PC(18:1(9Z)/18:1) \\
\hline 33 & 17.64 & 810.597 & $<0.001$ & 0.159 & $\mathrm{PC}(16: 0 / 22: 4)$ \\
\hline 34 & 17.65 & 760.5834 & 0.001 & 0.135 & $\mathrm{PC}(16: 0 / 18: 1)$ \\
\hline 35 & 13.58 & 813.6794 & 0.006 & 0.043 & SM (d16:1/26:1) \\
\hline 36 & 17.65 & 758.5666 & 0.034 & 0.015 & $\operatorname{PC}(16: 2 / 18: 0)$ \\
\hline
\end{tabular}

The $p$-value was calculated from Student's $t$ test. FC (fold change), defined as normalized peak area of metabolites in treatment group/control group. 
Table 3: Primary functional classification on differentially expressed proteins by iTRAQ

\begin{tabular}{|c|c|c|c|c|}
\hline Protein name & Accession & Fold change $^{a}$ & Trends $^{b}$ & $p$-value \\
\hline \multicolumn{5}{|l|}{ Glycolysis } \\
\hline $\begin{array}{l}\text { Katanin p60 ATPase-containing subunit A-like } \\
2\end{array}$ & E1BAN2 & $1.329+0.059$ & + & 0.005 \\
\hline L-lactate dehydrogenase B & B0JYN3 & $1.390+0.152$ & + & 0.021 \\
\hline Phosphoglycerate kinase & Q58DJ6 & $1.307+0.023$ & + & 0.079 \\
\hline Pyruvate kinase 1 & Q1JPG7 & $1.250+0.069$ & + & 0.083 \\
\hline Triosephosphate isomerase & Q5E956 & $0.639+0.052$ & - & 0.028 \\
\hline \multicolumn{5}{|l|}{ Pentose phosphate pathway } \\
\hline 6-phosphogluconate dehydrogenase & Q3ZCI4 & $1.420+0.143$ & + & 0.087 \\
\hline Flavin reductase (NADPH) & P52556 & $1.215+0.077$ & + & 0.012 \\
\hline Ribose-phosphate pyrophosphokinase 1 & Q2HJ58 & $1.343+0.063$ & + & 0.007 \\
\hline \multicolumn{5}{|l|}{ Oxidative reduction } \\
\hline Catalase & P00432 & $1.183+0.051$ & + & 0.032 \\
\hline Glutathione S-transferase P & P28801 & $1.472+0.118$ & + & 0.051 \\
\hline Lactoylglutathione lyase & A4FUZ1 & $1.252+0.130$ & + & 0.063 \\
\hline Peroxiredoxin-2 & Q9BGI3 & $1.292+0.088$ & + & 0.038 \\
\hline Peroxiredoxin-6 & O77834 & $1.483+0.124$ & + & 0.004 \\
\hline Superoxide dismutase $[\mathrm{Cu}-\mathrm{Zn}]$ & F1MNQ4 & $1.392+0.173$ & + & 0.024 \\
\hline Thioredoxin & G8JKZ8 & $1.503+0.097$ & + & 0.009 \\
\hline \multicolumn{5}{|l|}{ Others } \\
\hline $26 \mathrm{~S}$ protease regulatory subunit 7 & Q5E9F9 & $1.230+0.112$ & + & 0.045 \\
\hline Alpha-hemoglobin-stabilizing protein & Q865F8 & $1.321+0.076$ & + & 0.099 \\
\hline Hemoglobin, theta 1 & A1A4Q7 & $0.467+0.074$ & - & 0.017 \\
\hline Anion exchange protein & Q9XSW5 & $1.203+0.074$ & + & 0.052 \\
\hline Calmodulin & P62157 & $1.669+0.389$ & + & 0.057 \\
\hline Erythrocyte membrane protein band 4.2 & B0JYP3 & $1.212+0.104$ & + & 0.036 \\
\hline Tropomyosin alpha-3 chain & Q5KR47 & $1.169+0.082$ & + & 0.050 \\
\hline 14-3-3 protein beta/alpha & A0A140T894 & $1.238+0.095$ & + & 0.025 \\
\hline Acylamino-acid-releasing enzyme & P80227 & $1.324+0.165$ & + & 0.038 \\
\hline Acyl-CoA-binding domain-containing protein 7 & Q3SZF0 & $1.312+0.083$ & + & 0.012 \\
\hline $\begin{array}{l}\text { Serine/threonine-protein phosphatase } 2 \mathrm{~A} 65 \\
\mathrm{kDa} \text { regulatory subunit A alpha isoform }\end{array}$ & Q32PI5 & $1.459+0.171$ & + & 0.006 \\
\hline Alpha-2-macroglobulin & R9QSM8 & $1.673+0.372$ & + & 0.062 \\
\hline $\begin{array}{l}\text { ANTIGONDADOTROPIC DECAPEPTIDE, } \\
\text { AGD }\end{array}$ & Q9TRC1 & $3.996+2.332$ & + & 0.028 \\
\hline CD58 protein & A7YW37 & $1.806+0.454$ & + & 0.030 \\
\hline Coactosin-like protein & Q2HJ57 & $1.282+0.162$ & + & 0.076 \\
\hline Complement C3 & G3X7A5 & $1.751+0.295$ & + & 0.050 \\
\hline & & & & (Continued) \\
\hline
\end{tabular}




\begin{tabular}{lllcc}
\hline Protein name & Accession & Fold change & Trends ${ }^{\mathbf{b}}$ & $\boldsymbol{p}_{\text {-value }}$ \\
\hline Fatty acid-binding protein, epidermal & P55052 & $1.196+0.078$ & + & 0.026 \\
FGG protein/Fibrinogen gamma-B chain & P12799 & $1.708+0.287$ & + & 0.016 \\
Fibrinogen beta chain & P02676 & $1.666+0.446$ & + & 0.087 \\
Heat shock protein HSP 90-alpha & Q76LV2 & $1.194+0.104$ & + & 0.048 \\
Malate dehydrogenase, cytoplasmic & Q3T145 & $1.367+0.095$ & + & 0.020 \\
N(G), N(G)-dimethylarginine & Q3SX44 & $1.207+0.081$ & + & 0.041 \\
dimethylaminohydrolase 2 & Q3SZC4 & $1.232+0.068$ & + & 0.048 \\
NSFL1 cofactor p47 & Q17QX0 & $1.325+0.090$ & + & 0.003 \\
Nudix (Nucleoside diphosphate linked moiety & Q861V5 & $1.253+0.123$ & + & 0.051 \\
X)-type motif 5 & Q3ZBF7 & $1.177+0.090$ & + & 0.079 \\
Peptidyl-prolyl cis-trans isomerase & Q3T0Y5 & $1.521+0.214$ & + & 0.010 \\
Prostaglandin E synthase 3 & Q58DU5 & $1.266+0.050$ & + & 0.008 \\
Proteasome subunit alpha type-2 & Q5E987 & $1.228+0.097$ & + & 0.035 \\
Proteasome subunit alpha type-3 & Q3T108 & $1.368+0.160$ & + & 0.036 \\
Proteasome subunit alpha type-5 & A1L546 & $1.807+0.271$ & + & 0.003 \\
Proteasome subunit beta type-4 & Q3MHF7 & $1.384+0.137$ & + & 0.016 \\
Nucleolar protein NOP52 & G3X757 & $1.114+0.079$ & + & 0.092 \\
S-methyl-5'-thioadenosine phosphorylase & Q3MHL6 & $1.407+0.186$ & + & 0.047 \\
Transitional endoplasmic reticulum ATPase & A3KMV2 & $1.235+0.042$ & + & 0.028 \\
TSC22 domain family protein 1 & & & + \\
UV excision repair protein RAD23 homolog A & & & + \\
\hline
\end{tabular}

a Fold change: the ratios for the tags in the gossypol-treated group/tags in the control group.

$\mathrm{b}+$ : increased abundance; -: decreased abundance.

13 being elevated and 23 decreased in the gossypoltreated group as compared with the control group (Table 2). The 36 metabolites were classified into fatty amides (nine metabolites), phosphosphingolipids (three metabolites), glycerophosphoserines (two metabolites), glycerophosphocholines (six metabolites), and others (16 metabolites). The source dataset are presented in the Supplementary Material 2.

\section{Cross-validation of the metabolomics results with proteomics analysis}

To verify the metabolomics results, proteomics analysis was conducted to study the abundance change of the enzymes involved in the pathways of interest. A summary of the proteomics analysis results is presented in Supplementary Material 3. A total of 185 proteins were identified, of which 56 sequences were differentially expressed between the two groups with the criteria $p$ value of 0.1 . Then, these proteins were categorized based on their biological process and summarized in Table 3. Most enzymes involved in glycolysis, pentose phosphate pathway (PPP), and oxidative reduction were increased in abundance, except for triosephosphate isomerase 1, which catalyzes the transformation between glyceraldehydes3-phosphate and dihydroxyacetone phosphate, was decreased in abundance. Some other proteins including: membrane proteins, proteasomes, anion exchange protein, calmodulin, heat shock protein were also increased in abundance by gossypol treatment.

\section{Confirmation of proteomics analysis by enzyme activity assay}

Because both the metabolomics and proteomics results consistently indicated that the oxidationreduction is one of the most responsive pathways to the gossypol treatment, activity assays were performed for 6-phosphogluconate dehydrogenase, NADHP, glutathione S-transferase, and glutathione reductase. As shown in Figure 4, the activities of 6-phosphogluconate dehydrogenase, glutathione S-transferase, and glutathione reductase in the gossypol-treated group were all higher than those in the control group $(p=0.023,0.019$ and 
0.031, respectively). We also measured the concentrations of NADPH using commercial kits and found that the levels of NADPH in the gossypol-treated group were increased $(p=0.072)$. These results showed a strong correlation with the proteomics data.

\section{Integrating metabolomics and proteomics pathway analysis}

Differential metabolites were chosen for pathway analysis using MetaboAnalyst 3.0 [22] and grouped manually (Figures 5 and 6). It's showed that energy metabolism (glycolysis and the PPP), glutathione metabolism, and amino acid metabolism, among others were most profoundly affected by gossypol treatment. Glycolysis input compounds were reduced based on our detection of decreased glucose and its intermediate products glucose-6-phospahte $(\mathrm{FC}=0.778)$ and fructose6 -phosphate $(\mathrm{FC}=0.700)$. While the distal glycolysis products including pyruvate and lactate showed no changes. PPP was activated based on evidence of the accumulation of its end product, ribose-5-phosphate. We also observed decreases in glutathione disulfide levels, and the increased catabolites of glutathione, cysteine. The differential metabolites identified by UPLCQTOF also included phospholipids. Because mature $\mathrm{RBC}$ contains no cellular organelles and cannot utilize fatty acids to synthesize lipids, we suspect that these differential phosphatidycholine, phosphatidylserine, and sphingomyelin are likely from either the cell membrane, or the reticulocyte.

We further mapped the enzymes involved in the three pathways of interest (Figure 6). The abundance of glycolytic enzymes including enolase 1, phosphofructokinase, glyceraldehydes-3-phosphate dehydrogenase, and phosphoglycerate mutase 2 were not affected by gossypol. Triosephosphate isomerase 1 contents however, were decreased in the gossypol-treated group and this may cause the accumulation of dihydroxy acetone phosphate. In addition, L-lactate dehydrogenase, phosphoglycerate kinase, and pyruvate kinase 1 were elevated in the gossypol group, this may contribute to the unchanged levels of products in the distal of glycolysis, whereas the glycolysis input was decreased. Enzyme catalyzing the glucose-6-phosphate in the entrance step of the PPP, 6-phosphogluconate dehydrogenase, was upregulated and increased activity, and this was in good agreement with the increases of the end products of PPP,
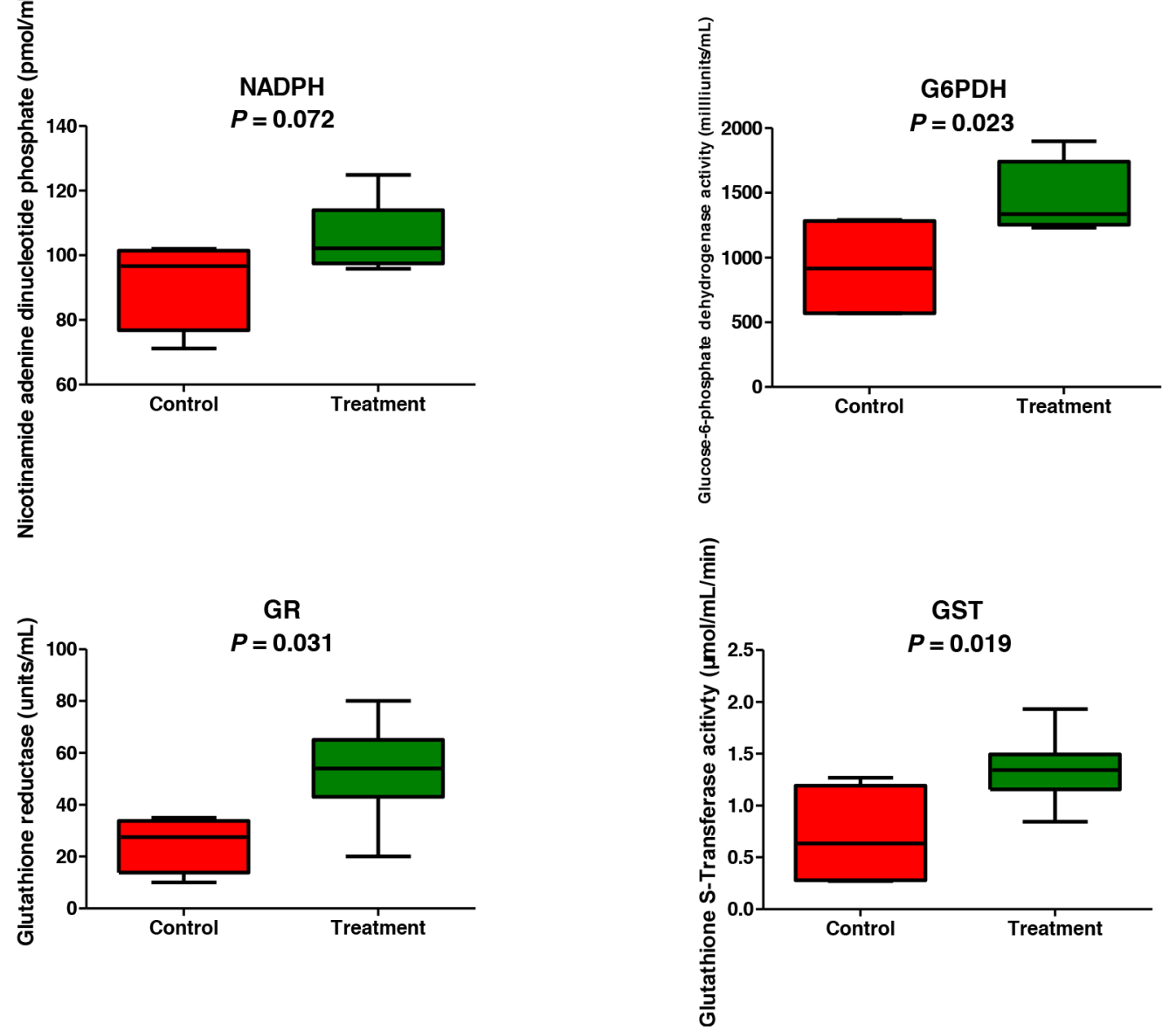

Figure 4:Confirmation of metabolomics and proteomics results by assaying oxidation-reduction enzymes activities. 


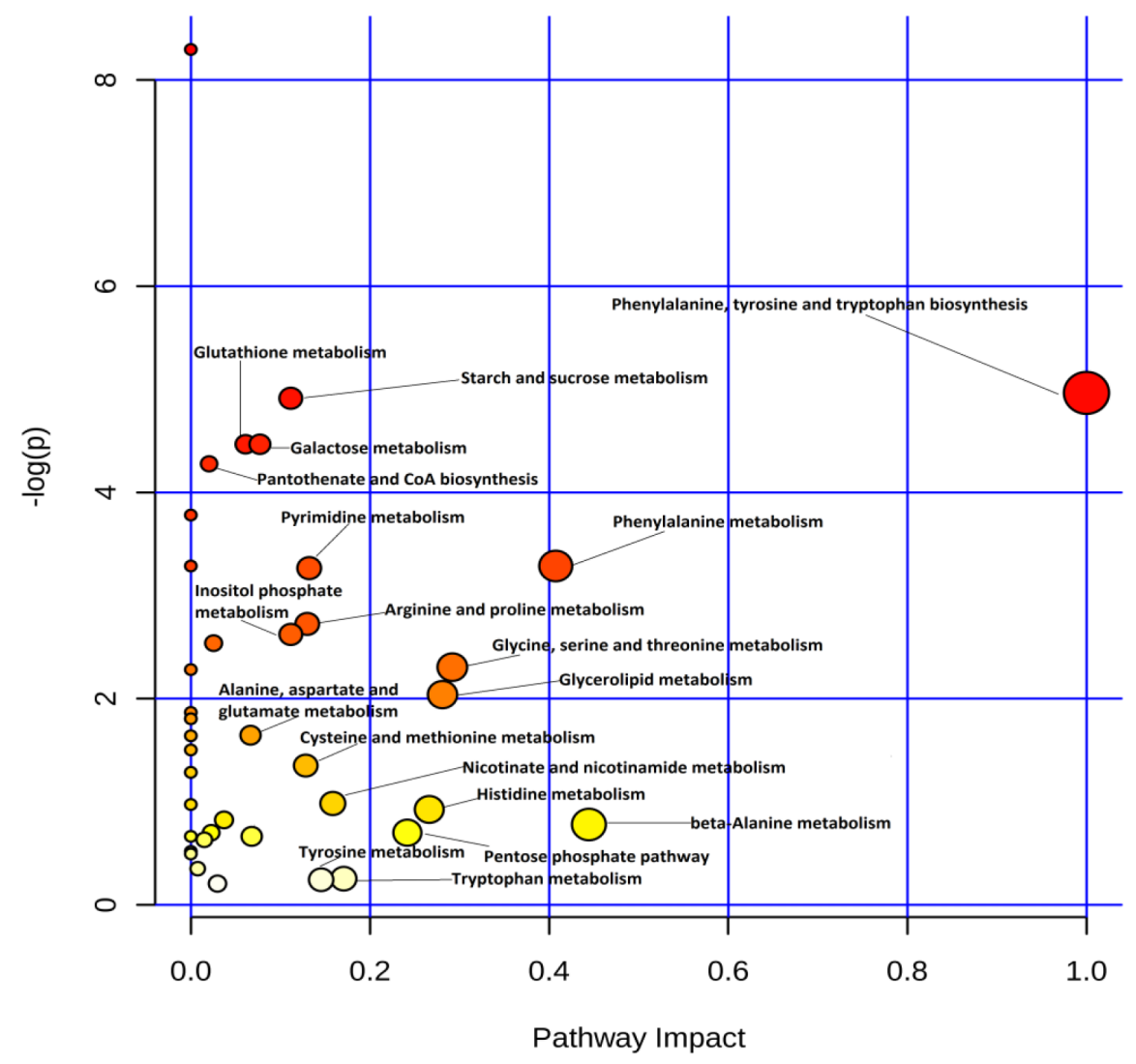

Figure 5: Pathway analysis of differential metabolites identified in red blood cells of cows from the control and gossypol-treated groups using MetaboAnalyst 3.0.

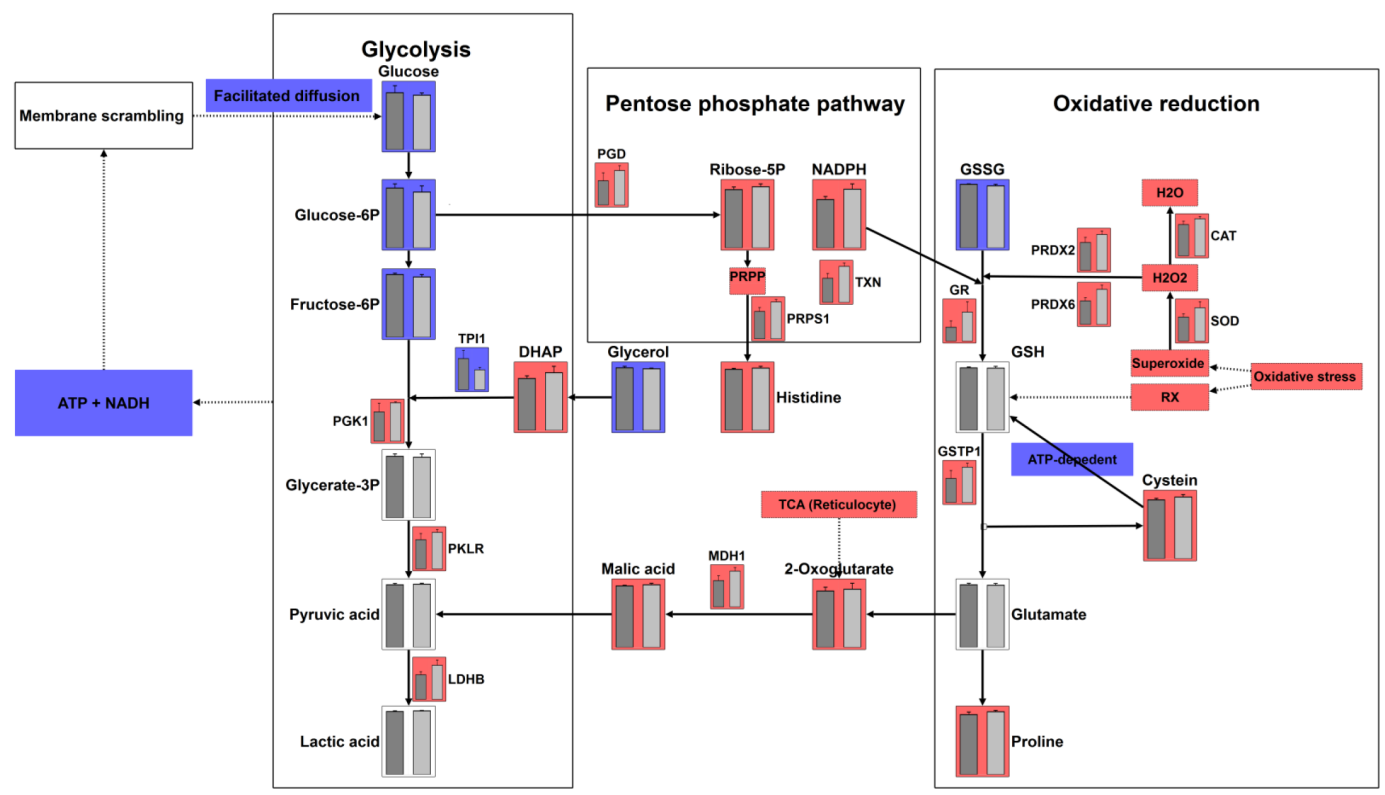

Figure 6: Integrating metabolomics and proteomics pathways: glycolysis, pentose phosphate pathway, oxidative reduction pathways. Red colored bar represents an increased abundance of metabolites or enzymes in the gossypol-treated group. Blue colored bar represents a decreased abundance of metabolites or enzymes in the gossypol-treated group. 
reducing power NADPH and ribose-5-phosphate. The elevated NADPH level was along with the increased glutathione reductase activity, which cooperated with each other to reduce glutathione disulfide to glutathione, and this was in line with the decreases of the glutathione disulfide. The glutathione S-transferase $\mathrm{P}$ was up-regulated and increased activity in the gossypol group, which may result in the increases of its catalysate, cysteine. Other oxidative reduction enzymes including, catalase, peroxiredoxin-2/6, thioredoxin and superoxide dismutase were all up-regulated, indicating an enhanced ability of antioxidation to combat the increased oxidative stress induced by gossypol treatment.

\section{DISCUSSION}

This study provided new insight into the RBC state at the molecular level in response to the hemolytic toxicity of gossypol through multi-omics analysis. Our hypothesis was formulated based on previous in vitro evidence showing that gossypol treatment induced suicidal erythrocyte death by stimulating $\mathrm{Ca}^{2+}$ entry [6] The mechanism on $\mathrm{Ca}^{2+}$ triggering cell death includes (a) activation of $\mathrm{Ca}^{2+}$-sensitive $\mathrm{K}^{+}$channels [11], the opening of $\mathrm{K}^{+}$channels results in $\mathrm{K}^{+}$exit, leading to cell membrane hyperpolarization, $\mathrm{Cl}^{-}$exit, and loss of cellular $\mathrm{KCl}$ with osmotically obliged water [12]; (b) leads to cell-membrane scrambling, with subsequent phosphatidylserine exposure at the erythrocyte surface [23]. To complement with previous in vitro data elucidating gossypol-induced eryptosis based on cation-homeostasis dysregulation [6], we used both metabolomics and proteomics approaches in gaining additional pieces of evidence for the mechanisms on hemolytic toxicity of gossypol in $\mathrm{RBC}$ in vivo. In our study, increased hemolysis was observed, as the $\mathrm{MCH}$ and $\mathrm{MCV}$ were decreased and osmotic fragility was increased in the gossypol-treated group. This initial observation inspired us to take a global metabolomics approach on RBCs to screen changes of relevantly responsive pathways. We found that the most changed pathways to gossypol treatment were three associated pathways including glycolysis, PPP, and oxidative reduction pathways. Consistent results were also obtained in our proteomics analysis and targeted-based enzymes activities assay, and both metabolites and protein data sets indicated that there was a good causal correlation between the abundance of enzymes involved in the pathways and the alteration of metabolome, although the expression of some glycolytic enzymes seems regulated by dynamic and complex mechanisms.

Gossypol binds to mineral elements, and iron was demonstrated to be the most efficient antidote to gossypol toxicity [14]. Thus, gossypol traps iron, a key player in hemoglobin generation, and may explain the decreased $\mathrm{MCH}$ in the gossypol-treated group. MCV and osmotic fragility are indicators of eryptosis and hemolysis.
The decreased MCV and increasing osmotic fragility observed here agreed well with previous studies reporting that treatment of gossypol resulted in RBC membrane scrambling and cell shrinkage in vitro [6] and increased osmotic fragility in vivo $[9,10]$. Additionally, our results support the notion that eryptosis and hemolysis were accelerated in vivo by gossypol treatment.

The differential metabolites identified from both GC-MS and UPLC-QTOF were analyzed to determine their relevant pathways that were subsequently grouped manually. Lower levels of glucose and its intermediate products, including glucose-6-phosphate and fructose-6phosphate, were initially observed in the gossypol-treated group. Glucose is the main energy source in RBC and enters RBC from circulating blood through facilitated diffusion. The disruption of the RBC membrane as a result of gossypol treatment was previously reported [6]; therefore, our observation of altered intracellular glucose levels agrees with those findings, although more evidence is needed to clarify the exact mechanisms involved. One of the most important biological roles of glycolysis is the production of ATP under anaerobic respiration, which is mainly used to maintain cation homeostasis. Energy depletion was also reported a stimulator of eryptosis [24]. Therefore, the reduction of glycolysis input may in turn exacerbate eryptosis and increase hemolysis. Furthermore, the regulation of glycolysis pathway was independently confirmed by proteomics results. A total of 8 glycolytic enzymes were identified, of which, the abundance of four, including phosphofructokinase, glyceraldeydes-3phosphate dehydrogenase, phosphoglycerate mutase 2, and enolase 1 were not changed, whereas three of them, including pyruvate kinase 1, phosphoglycerate kinase, and L-lactate dehydrogenase were increased in abundance and one enzyme, triosephosphate isomerase 1 was decreased in abundance. The expression of glycolytic enzymes was partly in line with the metabolites alteration including accumulated dihydroxy acetone phosphate and unchanged glycerate-3-phosphate, pyruvic acid, and lactic acid levels, decreased input metabolites of glycolysis. First of all, the low level of input metabolites of glycolysis observed in this study is consistent with the elevated PPP pathway evidenced with an increased key PPP metabolomic product and some key enzymes involved in PPP pathway. Secondly, since some key metabolic enzymes (e.g. pyruvate kinase) were allosterically regulated by the relevant metabolites through either feedforward stimulation or feedback inhibition, we suspect that the increased expression of three enzymes in distal steps of glycolysis may be the result of a compensation effect to the decreasing of glycolysis input and potential low concentration of ATP which would allow for maintenance of normal level of the metabolites and ATP in those distal steps. This was consistent with what we found in metabolomics data with unchanged metabolites in the distal steps of glycolysis. It should be pointed out that 
as mature RBCs lose capability of protein synthesis, we anticipate that reticulocytes may play a role in the compensation process, which was documented in other RBC disease [7]. A physiological response to compensate for anemia caused by hemolysis is increased reticulocyte production. Reticulocytes still preserve cytoplasmic organelles and are thus capable of protein synthesis. Several enzymes, including hexokinase, pyruvate kinase, and aldolase, display much higher activity in reticulocytes and are often referred to as the age-related enzymes [25]. Moreover, the post-translational modifications of the glycolytic enzymes may play a role in the RBCs' response to gossypol-induced oxidative stress, as it has been previously shown that activation of the PPP in RBCs by oxidative stress is at least in part mediated by reversible/ irreversible oxidation of functional cysteine and histidine of glyceraldehyde 3-phosphate dehydrogenase [26].

Despite energy metabolism, RBCs also need reducing status generated from glycolysis and PPP to effectively perform its normal function. One type of reducing source is through the PPP, wherein NADP+ is converted into NADPH. This NADPH is used in the glutathione metabolism pathway to reduce glutathione disulfide to glutathione, which is the major reducing agent for RBC [7]. In this study, the products of PPP, ribose5-phosphate and NADPH both were increased in the gossypol-treated group as detected by the metabolomics, proteomics, and targeted-based assay. This result indicated that more glucose entered into the PPP in response to gossypol treatment. Ratio of glycolysis/PPP flux constitutes an indirect parameter used to assess increased oxidative stress in RBC. Under normal steady state conditions, $92 \%$ of glucose is metabolized via glycolysis and $8 \%$ via the PPP [27]. Oxidation conditions will result in additional glucose molecules being metabolized through the PPP, which decreases the ratio of glycolysis/ PPP flux [27, 28]. Thus, the decreased glycolysis input and the activation of the PPP may imply gossypol-induced oxidative stress, a property that was well documented before, which showed that gossypol exhibited pro-oxidant properties according to in vitro and in vivo studies based on elevations in reactive oxygen species with increased malondialdehyde production, and decreased glutathione concentrations $[15,16,29,30]$. Further studies performing tracing experiments by incubating RBCs with $13 \mathrm{C} 1,2$ or $13 \mathrm{C} 1$, 2, 3-glucose to confirm the activation of glycolysis vs PPP as observed in this study would be of great value. Along with the elevated NADPH, the glutathione reductase activity was increased, this could reduce more glutathione disulfide to glutathione and evidenced by lower level of the glutathione disulfide in the gossypol group. The enhanced transformation from glutathione disulfide to glutathione indicated an increased protective effect of glutathione in response to gossypol-induced stress, and was also confirmed by the increased abundance of glutathione S-transferase $\mathrm{P}$ in our proteomics data and the increased activity of glutathione S-tranferase in targeted-based assay, which catalyzes the conjugation of glutathione to a wide number of hydrophobic electrophiles. As glutathione transferase was up-regulated and activity increased, we also detected accumulation of the catabolites of glutathione, cysteine. Additionally, the increased cysteine may also result from the depression of de novo synthesis of glutathione, which is ATP dependent, as we demonstrated the decreases of glycolysis input. In spite of glutathione metabolism, other oxidative reduction enzymes, including superoxide dismutase, catalase, peroxiredoxin-2, peroxiredoxin-6, and thioredoxin were up-regulated in response to gossypol treatment. However, these complements did not appear sufficient to effectively protect RBC from oxidative stress, whereas the MCV and osmotic fragility were still significantly altered as shown at the beginning of this study.

Besides metabolites and enzymes involved in glycolysis, PPP, and oxidative reduction pathways, 2-oxoglutarate and malic acid were also identified in this study and in elsewhere [19], which may explained by transaminase activity and cytosolic malate dehydrogenase activity [31] or may come from the reticulocytes. Both 2-oxoglutarate and malic acid $(\mathrm{FC}=1.286, p=0.168)$ levels were increased in response to gossypol treatment, along with the increased abundance of the malate dehydrogenase. This may imply an enhanced TCA in the reticulocytes to supply more ATP to combat decreased ATP generation from glycolysis in mature RBC. The differential metabolites identified also included lipids; however, lipid synthesis is active in reticulocytes and suppressed in mature RBC [32]. Therefore, the differential lipids identified in this study may belong to the $\mathrm{RBC}$ membrane or from reticulocytes. The alteration of lipid concentrations in the gossypol-treated group may be an indicator that the RBC membrane was disrupted as a result of gossypol treatment. The reduction in six phosphatidycholine species, two phosphatidylserine species, and two sphingomyelin species in the gossypoltreated group may have indicated the decreased membrane fluidity, resulting in an increased osmotic fragility attributed to hemolysis.

In conclusion, we characterized the RBC state at molecular level in response to the hemolytic toxicity of gossypol by performing multi-omics analysis. Acceleration of hemolysis was observed after gossypol treatment and subsequent altered pathways were identified by untargeted metabolomics and further cross-verified by comparative proteomics, which later validated by targetedbased enzyme activity assay. Our both omics data sets on RBCs showed that glycolysis input was decreased, the PPP products and metabolism were elevated, and the oxidative reduction metabolites for antioxidation reactions were remarkably increased by gossypol treatment. Both metabolomics and proteomics data revealed a good causal correlation between enzymes involved in the specific 
pathways and the concordant alteration of metabolome. Through the global multi-omics analysis, we conclude that both glycolysis and PPP pathways responsible for essential energy metabolism and maintenance of reducing agents, along with the oxidative reduction pathways for antioxidation reactions, were involved in $\mathrm{RBC}$ in response to the hemolytic toxicity of gossypol. Our results may benefit further studies for developing gossypol as an anticancer drug.

\section{MATERIALS AND METHODS}

\section{Chemicals and reagents}

Gossypol-acetic acid (98\% purity) was provided by Yangling Ciyuan Biotechnology Co., Ltd (Shanxi, China). Methanol, formic acid, acetonitrile, chloroform, and other chemicals were of high-performance liquid chromatography grade. Amino acid internal standard, methoxyamine hydrochloride, pyridine, triethylammonium bicarbonate, and iodoacetamide were purchased from Sigma-Aldrich (St. Louis, MO, USA). Bis (trimethylsilyl) trifluoroacetamide (BSTFA) and trimethylchlorosilane (TMCS) were obtained from Regis Technologies (Morton Grove, IL, USA). Dithiothreitol was obtained from Affymetrix (Santa Clara, CA).

\section{Animal treatment, sample collection and RBC parameters determination}

Twelve gossypol-blank middle-lactating Holstein dairy cows were randomly divided into two groups: control group (four animals) and gossypol-treated group (eight animals). Animals in the gossypol-treatment group were administered with $29.58 \pm 3.22 \mathrm{mg}$ gossypol per $\mathrm{kg}$ body weight for 28 days, to reach $5-10 \mu \mathrm{M}$ plasma negative gossypol, which shown to be effective inhibited cells proliferation in vitro study [4]. All animals were fed a gossypol-free diet and had ad libitum access to feed and water. Gossypol was transferred into capsules and administered to the animals twice a day. Animals in the control group were administered empty capsules.

On day 28, blood samples were collected from the jugular vein into EDTA-2K and sodium heparin-coated Vacutainer tubes (Greiner Bio-one GmbH, Kremsmünster, Austria). Blood anticoagulated by EDTA-2K were used for RBC parameters determination, and blood anticoagulated by sodium heparin were used for osmotic fragility determination and $\mathrm{RBC}$ separation. To separate $\mathrm{RBC}$, plasma was removed by centrifugation at $1450 \mathrm{~g}$ for 10 min. RBC pellets were washed with $0.9 \%$ saline, and the supernatant was removed by centrifugation at $1450 \mathrm{~g}$ for 5 min. The wash process was repeated 3 times. The up layer leukocyte and platelet were discarded, and the lower layer RBCs were stored in liquid-nitrogen prior to analysis. The animal protocol was approved by the Animal Care and Use
Committee of the Institute of Animal Science, Chinese Academy of Agricultural Sciences.

$\mathrm{RBC}$ parameters, including the amounts of $\mathrm{RBC}$, $\mathrm{HGB}$, mean corpuscular $\mathrm{HGB}$ and volume (MCH and $\mathrm{MCV}$, respectively), and mean corpuscular $\mathrm{HGB}$ concentration (MCHC) were determined using a Mindray BC-2800Vet auto hematology analyzer (Shenzhen Mindray Bio-Medical Electronics Co., Ltd., Shenzhen, China). Osmotic fragility determination was performed according to a previously described method [33] by measuring the percentage of hemolyzed RBC in a solution of $0.8 \% \mathrm{NaCl}$-phosphate buffer, which was compared with that observed in distilled water (100\% hemolysis).

\section{Metabolomics analysis}

A 50-mg sample of RBC and $950 \mu \mathrm{L}$ of methanol/ chloroform/water solvent (volumetric ratio: 700:200:50) were successively added to an Eppendorf tube. The cell mixture was sonicated for $1 \mathrm{~min}$ on ice and incubated at $-20^{\circ} \mathrm{C}$ for $1 \mathrm{~min}$. This process was repeated five times. The mixture was then stored at $-40^{\circ} \mathrm{C}$ for $24 \mathrm{~h}$ prior to centrifugation at $16,000 \mathrm{~g}$ for $15 \mathrm{~min}$ at $4^{\circ} \mathrm{C}$, followed by addition of $250 \mu \mathrm{L}$ of the mixture to a $\mathrm{GC}$ vial containing $10 \mu \mathrm{L}$ of internal standards $(0.05 \mathrm{mg} / \mathrm{mL}$ of $13 \mathrm{C} 3-15 \mathrm{~N}-$ L-alanine, 13C5-15N-L-valine, 13C6-15N-L-leucine, and $13 \mathrm{C} 6-15 \mathrm{~N}-\mathrm{L}-$ isoleucine). The mixture was dried under a gentle nitrogen stream, and $30 \mu \mathrm{L}$ of $20 \mathrm{mg} / \mathrm{mL}$ methoxyamine hydrochloride in pyridine was added to the vial with the dry residue. The resulting mixture was vortexed vigorously for $30 \mathrm{~s}$ and incubated at $37^{\circ} \mathrm{C}$ for $90 \mathrm{~min}$. BSTFA ( $30 \mu \mathrm{L}$, with $1 \%$ TMCS $)$ was added to the mixture and derivatized at $70^{\circ} \mathrm{C}$ for $60 \mathrm{~min}$ prior to performing GC-MS metabolomics analysis. For UPLCQTOF analysis, samples were sonicated as the same method described above for the GC-MS analysis. Then, the mixture was centrifuged at $16,000 \mathrm{~g}$ for $10 \mathrm{~min}$ at $4^{\circ} \mathrm{C}$. The supernatant $(300 \mu \mathrm{L})$ was transferred to new tubes and dried under nitrogen gas, and the residue was resuspended in $100 \mu \mathrm{L}$ acetonitrile/water (volumetric ratio: 50:50) solution for UPLC-QTOF analysis.

Metabolomics analysis was performed using an Agilent 7890A GC system coupled to an Agilent 5975C inert MSD system (Agilent Technologies, Santa Clara, CA, USA). A HP-5ms fused-silica capillary column (30 $\mathrm{m} \times 0.25 \mathrm{~mm} \times 0.25 \mu \mathrm{m}$; Agilent J\&W Scientific, Folsom, CA, USA) was used to separate the derivatives. Helium was used as a carrier gas at a constant flow rate of 1 $\mathrm{mL} / \mathrm{min}$. Injection volume was $1 \mu \mathrm{L}$ in splitless mode, and the solvent-delay time was $6 \mathrm{~min}$. The initial oven temperature was held at $70^{\circ} \mathrm{C}$ for $2 \mathrm{~min}$, ramped to $160^{\circ} \mathrm{C}$ at a rate of $6^{\circ} \mathrm{C} / \mathrm{min}$, then to $240^{\circ} \mathrm{C}$ at a rate of $10^{\circ} \mathrm{C} /$ $\min$, to $300^{\circ} \mathrm{C}$ at a rate of $20^{\circ} \mathrm{C} / \mathrm{min}$, and finally held at $300^{\circ} \mathrm{C}$ for $6 \mathrm{~min}$. The temperatures of the injector, transfer line, and electron-impact ion source were set to $250^{\circ} \mathrm{C}$, $290^{\circ} \mathrm{C}$, and $230^{\circ} \mathrm{C}$, respectively. The impact energy was 
$70 \mathrm{eV}$, and data were collected in full-scan mode $(\mathrm{m} / \mathrm{z}$ 50-600). AMDIS software was used to deconvolute mass spectra from raw GC-MS data, and the mass spectra were automatically matched using an in-house standard library, including retention time and mass spectra, the Golm Metabolome Database (http://gmd.mpimp-golm.mpg. de/), and the Agilent Fiehn GC/MS metabolomics RTL library (Agilent Technologies). Peak picking, alignment, deconvolution, and further processing of raw GC-MS data were performed according to previously described methods [34].

A 1290 series UPLC (Agilent Technologies) was used for separation on a ACQUITY UPLC HSS T3 columns $(100 \mathrm{~mm} \times$ i.d. $2.1 \mathrm{~mm})$ with $1.7-\mu \mathrm{m}$ particles at $25^{\circ} \mathrm{C}$ (Waters Corporation, Milford, MA, USA). The mobile phase consisted of formic acid/water $(0.1 \%$, solution $\mathrm{A})$ and formic acid/acetonitrile $(0.1 \%$, solution $\mathrm{B})$, and the flow rate was set at $0.3 \mathrm{~mL} / \mathrm{min}$. A gradient elution condition was applied as follows: $0-1.5 \mathrm{~min}, 1 \% \mathrm{~B} ; 13$ min, 99\% B; $16.5 \mathrm{~min}, 99 \% \mathrm{~B} ; 16.6 \mathrm{~min}, 1 \% \mathrm{~B}$ maintain to $20 \mathrm{~min}$. MS data were collected using a TripleTOF 6600 (AB Sciex, concord, ON, Canada). Electrospray ionization was used as the ionization source, and analysis was performed in positive mode with a source temperature of $300^{\circ} \mathrm{C}$. The scan was performed in MS1 range from 60 Da to 1000 Da. Parent ions were selected automatically using the IDA model in MS2. Collision energy was set at $35 \pm 15$ ev. Metabolites according to UPLC-QTOF were identified by matching the MS1 and MS2 data in Lipidblast [35]. UPLC-QTOF raw data were transferred into mzXML format using the MSConvert software from Proteowizard [36]. Deconvoluted MS1 data (parent ions) and corresponding MS2 data (daughter ions) were obtained after peak matching, alignment, and retentiontime correction using XCMS data-analysis software [37]. Isotope peaks and adduct ions were analyzed using the CAMERA package, and data were normalized against total peak intensities before performing univariate and multivariate statistical analysis.

\section{Sample preparation for proteomic analysis}

RBC proteins were obtained by homogenizing 100 $\mu \mathrm{L}$ RBC samples in $700 \mu \mathrm{L} 50 \mathrm{mM}$ triethylammonium bicarbonate buffer ( $\mathrm{pH} 8.5$ ) containing $6 \mathrm{M}$ urea. The homogenate was centrifuged at $2000 \mathrm{~g}$ for $15 \mathrm{~min}$. Protein concentration were quantified by the BSA method, and checked by SDS-PAGE. An aliquot $(100 \mu \mathrm{g})$ proteins was reduced in $100 \mu \mathrm{L}$ system containing $50 \mathrm{mM}$ dithiothreitol at $37^{\circ} \mathrm{C}$ for an hour. Cysteine residues were blocked with $10 \mu \mathrm{L}$ of $1 \mathrm{M}$ iodoacetamide for $30 \mathrm{~min}$ in the dark. The proteins were precipitated by acetone overnight and then digested using a trypsin (V511A, Promega) to substrate ratio of $50: 1$, at $37^{\circ} \mathrm{C}$ overnight. The digested peptides were labeled with iTRAQ 8-plex reagents following the manufacturer's instructions using 113-tags, 114-tag, 115- tag, and 116-tag, for control group, and 117-tag, 118tag, 119-tag, and 121-tag, for gossypol-treated group, respectively. After labeling efficiency assessment, the eight samples were combined and subjected to high $\mathrm{pH}$ reverse phase fractionation.

The high reverse phase chromatography was carried out using Agilent 1290 high performance LC system with an autosampler and UV detection. The pooled and labeled peptides were loaded onto a C18 column $(5 \mu \mathrm{m}, 4.6 \times 250$ $\mathrm{mm}$, Beijing TechMate Technology CO., LTD.) with $5 \mathrm{mM}$ ammonium formate, $2 \%$ acetonitrile in water as buffer $\mathrm{A}$ $(\mathrm{pH} 9.0)$ and $5 \mathrm{mM}$ ammonium formate, $10 \%$ water in acetonitrile as buffer $\mathrm{B}$ ( $\mathrm{pH} 9.0)$. The LC was performed using a gradient from 5 to $80 \%$ of buffer B in 50 min at a flow rate of $0.6 \mathrm{~mL} / \mathrm{min}$. Forty fractions were collected at 1 min intervals and pooled into a total of 8 fractions, based on UV absorbance at $214 \mathrm{~nm}$ and with a multiple fraction concatenation strategy. The 8 pooled fractions were dried for subsequent nanoLC-MS/MS analysis.

\section{Protein identification and quantitation}

Peptide fractions were reconstituted in $20 \mu \mathrm{L}$ of $0.1 \%$ formic acid, $2 \%$ acetonitrile in water and analyzed by TripleTOF 6600 (Sciex, Framingham, MA, USA). Peptide mixtures were injected into the capillary column $(0.075 \times 150 \mathrm{~mm})$ and separated by a $3 \mu \mathrm{m} \mathrm{C} 18$ column. Peptides were eluted with a liner gradient of $5-40 \%$ buffer B $(0.1 \%$ formic acid, $2 \%$ water, and $5 \%$ DMSO in acetonitrile) at a flow rate of $0.3 \mu \mathrm{L}$ for $90 \mathrm{~min}$. Buffer A consisted of $0.1 \%$ formic acid, $2 \%$ acetonitrile, $5 \%$ DMSO (dissolved in acetonitrile) in water. The full MS1 scans were performed at the range of $350-1,500 \mathrm{~m} / \mathrm{z}$ in $250 \mathrm{~ms}$. The 40 most abundant ions with multiple charge states were selected for higher energy collision and the scan range was between $100-1500 \mathrm{~m} / \mathrm{z}$. The MS/MS spectra were obtained and searched using MaxQuant (version 2.5.6.5, Martinsried, Germany) against Uniprot Bos taurus protein database containing 6,887 sequences. Mass tolerances for precursor ions and fragment ions were 0.1 $\mathrm{Da}$ and $40 \mathrm{ppm}$, respectively. The proteins and peptides were filtered with a false discovery rate $($ FDR $)<1 \%$. The enzyme parameter was limited to semi-tryptic peptides with a maximum miscleavage of 2 . Variable modification of oxidation of methionine residue was selected, and carbamidomethylated cysteine, iTRAQ 8-plex (K) and iTRAQ 8-plex (N-term) were set as static modification.

\section{Enzyme activity assay}

To confirm the results of proteomics analysis, the activities of four enzymes including 6-phosphogluconate dehydrogenase, flavin reductase (NADPH), glutathione $\mathrm{S}$-transferase, and glutathione reductase which involved in the pathway of interests, were all determined by commercial kits purchased from Sigma-Aldrich 
(St. Louis, MO, USA). In the 6-phosphogluconate dehydrogenase kit, glucose-6-phosphate is oxidized to generate a product, which is specifically detected by colorimetric $(450 \mathrm{~nm})$ assay. NADPH was determined by measuring a fluorescent product $\left(\lambda_{\mathrm{ex}}=535 / \lambda_{\mathrm{em}}=587\right.$ $\mathrm{nm})$ proportional to the amount of NADPH present. Glutathione S-transferase catalyzes the conjugation of L-glutathione to 1-Chloro-2, 4-dinitrobenzene through the thiol group of the glutathione. The reaction product absorbs at $340 \mathrm{~nm}$. The rate of increase in the absorption is directly proportional to the glutathione S-transferase activity in the sample. The activity of glutathione reductase was measured by the decrease in absorbance caused by the oxidation of NADPH at $340 \mathrm{~nm}$ (UV assay).

\section{Experimental design and statistical rationale}

The samples include four biological replicates for control group $(n=4)$ and eight biological replicates for gossypol-treated group $(n=8)$. Metabolome and proteome analyses were performed in randomized order to eliminate systematic biases. The normalized dataset was imported into MetaboAnalyst 3.0 [22] for principal component analysis (PCA) and partial least squares discriminant analysis (PLS-DA). Data from UPLCQTOF were interquantile range filtered. All data were auto scaled prior to multivariate analysis. Univariate analysis was performed to screen potential differential metabolites and proteins. Student's $t$ test was conducted to compare the differential metabolites between the two groups. A fold change (FC), defined as normalized peak area of metabolites in treatment group/control group, was calculated as one of the options for differential metabolite selection. For the iTRAQ-based proteomics data, abundance of proteins was compared bases on Student's $t$ test carried out on log2-transferred iTRAQ ratio. Attention should be paid to the criteria selected for the differential metabolites and proteins. Compared with living organisms and other cells containing organelles, mature RBC do not possess a strong ability to resist stress due to the lack of all organelles, if the alteration is out of its endurance, hemolysis happens. Besides, the major aim of the metabolomics analysis was to screen pathways of change, which were further cross-validated by iTRAQ-based proteomics data set and confirmed by targeted-based enzymatic assays. On the basis of above, the following criteria were used for differential metabolites selection: $p<0.05, \mathrm{FC}>1.4$ or $<0.67$ (inter error was defined as the value of $\mathrm{FC}$ at which $90 \%$ of all metabolites had no deviation from each other). Metabolites exhibiting changes between the control and gossypol-treated groups were selected for pathway analysis using MetaboAnalyst 3.0 [22]. And differential abundance of proteins and enzymes activities with $p$ $<0.1$ with what ratio cutoff were considered when integrating with metabolomics results.

\section{Abbreviations}

RBC, red blood cell; HGB, hemoglobin; $\mathrm{MCH}$, mean corpuscular hemoglobin; $\mathrm{MCV}$, mean corpuscular volume; MCHC, mean corpuscular hemoglobin concentration; PCA, principal component analysis; PLSDA, partial least squares discriminant analysis; FC, fold change; RSD, relative standard derivations; PPP, pentose phosphate pathway.

\section{Author contributions}

Chaohua Tang, Qingyu Zhao, and Junmin Zhang designed and supervised all the overall experiment. Chaohua Tang, Kai Zhang, and Tengfei Zhan conducted the animal experiments and collected the samples. Chaohua Tang, Qingshi Meng, and Sheng Zhang performed instruments and statistical analyses, and Chaohua Tang wrote the paper. All authors critically commented on the manuscript.

\section{CONFLICTS OF INTEREST}

All authors disclose no conflicts of interests relevant to this study.

\section{FUNDING}

This work was supported by a grant from the National Natural Science Foundation of China (31472123) and the Chinese Academy of Agricultural Science and Technology Innovation Project (CAASXTCX2016011-01).

\section{REFERENCES}

1. Wang A, Zhang J, Meng Y, Deng L, Lv Y, Li C, Wang J. Effects of different sources and levels of dietary gossypol on gossypol residues in plasma and milk of lactating cows. J Dairy Sci. 2012; 95: 5127-32. https://doi.org/10.3168/ jds.2011-4870.

2. Wang X, Howell CP, Chen F, Yin J, Jiang Y. Gossypol-a polyphenolic compound from cotton plant. Adv Food Nutr Res. 2009; 58: 215-63. https://doi.org/10.1016/ S1043-4526(09)58006-0.

3. Traynor AM, Kolesar J, Marnocha RM, Eikhoff JC, Alberti DB, Takebe N, Wilding G, Liu G, Schelman WR. (2011). A phase I study of R-(-)-gossypol (AT-101) in combination with cisplatin $(\mathrm{P})$ and etoposide $(\mathrm{E})$ in patients (pts) with advanced solid tumors and extensive-stage small cell lung cancer (ES-SCLC). America Society of Clinical Oncology Annual Meeting, pp. 3040.

4. Mcgregor N, Sargent E, Loberg R. AT101 induced apoptosis in $\mathrm{VCaP}$ androgen sensitive cells is inhibited by the presence of DHT. Cancer Res. 2007. 
5. Wang C, Yeung RTT. Gossypol and hypokalaemia. Contraception. 1985; 32: 237-52. https://doi. org/10.1016/0010-7824(85)90047-2.

6. Zbidah M, Lupescu A, Shaik N, Lang F. Gossypol-induced suicidal erythrocyte death. Toxicology. 2012; 302: 101-5. https://doi.org/10.1016/j.tox.2012.09.010.

7. van Wijk R, van Solinge WW. The energy-less red blood cell is lost: erythrocyte enzyme abnormalities of glycolysis. Blood. 2005; 106: 4034-42. https://doi.org/10.1182/ blood-2005-04-1622.

8. Barasa B, Slijper M. Challenges for red blood cell biomarker discovery through proteomics. Biochim Biophys Acta. 2014; 1844: 1003-10. https://doi.org/10.1016/j. bbapap.2013.10.002.

9. Mena H, Santos J, Huber J, Tarazon M, Calhoun M. The effects of varying gossypol intake from whole cottonseed and cottonseed meal on lactation and blood parameters in lactating dairy cows. J Dairy Sci. 2004; 87: 2506-18. https:// doi.org/10.3168/jds.S0022-0302(04)73375-5.

10. Mena H, Santos J, Huber J, Simas J, Tarazon M, Calhoun M. The effects of feeding varying amounts of gossypol from whole cottonseed and cottonseed meal in lactating dairy cows. J Dairy Sci. 2001; 84: 2231-9. https://doi. org/10.3168/jds.S0022-0302(01)74670-X.

11. Brugnara $\mathrm{C}$, de Franceschi L, Alper SL. Inhibition of $\mathrm{Ca}(2+)$-dependent $\mathrm{K}+$ transport and cell dehydration in sickle erythrocytes by clotrimazole and other imidazole derivatives. J Clin Invest. 1993; 92: 520-6. https://doi. org/10.1172/JCI116597.

12. Lang PA, Kaiser S, Myssina S, Wieder T, Lang F, Huber $\mathrm{SM}$. Role of $\mathrm{Ca} 2+$-activated $\mathrm{K}+$ channels in human erythrocyte apoptosis. Am J Physiol Cell Physiol. 2003; 285: C1553-60. https://doi.org/10.1152/ajpcell.00186.2003.

13. Gao M, Cheung KL, Lau IP, Yu WS, Fung KP, Yu B, Loo JF, Kong SK. Polyphyllin D induces apoptosis in human erythrocytes through $\mathrm{Ca} 2+$ rise and membrane permeabilization. Arch Toxicol. 2012; 86: 741-52. https:// doi.org/10.1007/s00204-012-0808-4.

14. Smith F, Clawson A. The effects of dietary gossypol on animals. J Am Oil Chem Soc. 1970; 47: 443-7. https://doi. org/10.1007/BF02632963.

15. Laughton MJ, Halliwell B, Evans PJ, Robin J, Hoult S. Antioxidant and pro-oxidant actions of the plant phenolics quercetin, gossypol and myricetin: effects on lipid peroxidation, hydroxyl radical generation and bleomycindependent damage to DNA. Biochem Pharmacol. 1989; 38: 2859-65. https://doi.org/10.1016/0006-2952(89)90442-5.

16. de Peyster A, Quintanilha A, Packer L, Smith MT. Oxygen radical formation induced by gossypol in rat liver microsomes and human sperm. Biochem Biophys Res Commun. 1984; 118: 573-9. https://doi. org/10.1016/0006-291X(84)91341-X.

17. Lang F, Abed M, Lang E, Föller M. Oxidative stress and suicidal erythrocyte death. Antioxid Redox Signal. 2014; 21: 138-53. https://doi.org/10.1089/ars.2013.5747.
18. Darghouth D, Koehl B, Madalinski G, Heilier JF, Bovee P, $\mathrm{Xu}$ Y, Olivier MF, Bartolucci P, Benkerrou M, Pissard S. Pathophysiology of sickle cell disease is mirrored by the red blood cell metabolome. Blood. 2011; 117: e57-e66. https:// doi.org/10.1182/blood-2010-07-299636.

19. D'Alessandro A, Nemkov T, Sun K, Liu H, Song A, Monte AA, Subudhi AW, Lovering AT, Dvorkin D, Julian CG, Kevil CG, Kolluru GK, Shiva S, et al. AltitudeOmics: red blood cell metabolic adaptation to high altitude hypoxia. J Proteome Res. 2016; 15: 3883-95. https://doi.org/10.1021/ acs.jproteome.6b00733.

20. Paglia G, D’Alessandro A, Rolfsson Ó, Sigurjónsson ÓE, Bordbar A, Palsson S, Nemkov T, Hansen KC, Gudmundsson S, Palsson BO. Biomarkers defining the metabolic age of red blood cells during cold storage. Blood. 2016; 128: e43-e50. https://doi.org/10.1182/ blood-2016-06-721688.

21. D'Alessandro A, Zolla L. Proteomic analysis of red blood cells and the potential for the clinic: what have we learned so far? Expert Rev Proteomics. 2017; 14: 243-52. https:// doi.org/10.1080/14789450.2017.1291347.

22. Xia J, Sinelnikov IV, Han B, Wishart DS. MetaboAnalyst 3.0 - making metabolomics more meaningful. Nucleic Acids Res. 2015; 43: W251-W7. https://doi.org/10.1093/ nar/gkv380.

23. Lang F, Gulbins E, Lerche H, Huber SM, Kempe DS, Föller M. Eryptosis, a window to systemic disease. Cell Physiol Biochem. 2008; 22: 373-80. https://doi. org/10.1159/000185448.

24. Klarl BA, Lang PA, Kempe DS, Niemoeller OM, Akel A, Sobiesiak M, Eisele K, Podolski M, Huber SM, Wieder T. Protein kinase $\mathrm{C}$ mediates erythrocyte "programmed cell death" following glucose depletion. Am J Physiol Cell Physiol. 2006; 290: C244-C53. https://doi.org/10.1152/ ajpcell.00283.2005.

25. Beutler E. The relationship of red cell enzymes to red cell life-span. Blood Cells. 1988; 14: 69-91.

26. Reisz JA, Wither MJ, Dzieciatkowska M, Nemkov $T$, Issaian A, Yoshida T, Dunham AJ, Hill RC, Hansen KC, D'Alessandro A. Oxidative modifications of glyceraldehyde 3-phosphate dehydrogenase regulate metabolic reprogramming of stored red blood cells. Blood. 2016; 128: e32-e42. https://doi.org/10.1182/blood-2016-05-714816.

27. Gevi F, D'Alessandro A, Rinalducci S, Zolla L. Alterations of red blood cell metabolome during cold liquid storage of erythrocyte concentrates in CPD-SAGM. J Proteomics. 2012; 76: 168-80. https://doi.org/10.1016/j. jprot.2012.03.012.

28. Messana I, Misiti F, el-Sherbini S, Giardina B, Castagnola M. Quantitative determination of the main glucose metabolic fluxes in human erythrocytes by $13 \mathrm{C}$ - and 1H-MR spectroscopy. J Biochem Biophys Methods. 1999; 39: 63-84. https://doi.org/10.1016/S0165-022X(99)00005-6.

29. Kovacic P. Mechanism of drug and toxic actions of gossypol: focus on reactive oxygen species and electron 
transfer. Curr Med Chem. 2003; 10: 2711-8. https://doi. org/10.2174/0929867033456369.

30. El-Sharaky A, Wahby M, El-Dein MB, Fawzy R, El-Shahawy I. Mutual anti-oxidative effect of gossypol acetic acid and gossypol-iron complex on hepatic lipid peroxidation in male rats. Food Chem Toxicol. 2009; 47: 2735-41. https://doi.org/10.1016/j.fct.2009.08.001.

31. D'Alessandro A, Nemkov T, Yoshida T, Bordbar A, Palsson BO, Hansen KC. Citrate metabolism in red blood cells stored in additive solution-3. Transfusion. 2017; 57: 32536. https://doi.org/10.1111/trf.13892.

32. Percy AK, Schmell E, Earles B, Lennarz W. Phospholipid biosynthesis in the membranes of immature and mature red blood cells. Biochemistry. 1973; 12: 2456-61. https://doi. org/10.1021/bi00737a014

33. Sharma B, Rai DK, Rai PK, Rizvi S, Watal G. (2010). Determination of erythrocyte fragility as a marker of pesticide-induced membrane oxidative damage. Advanced Protocols in Oxidative Stress II: Springer), pp. 123-8.
34. Gao XF, Pujos-Guillot E, Sebedio JL. Development of a quantitative metabolomic approach to study clinical human fecal water metabolome based on trimethylsilylation derivatization and GC/MS analysis. Anal Chem. 2010; 82: 6447-56. https://doi.org/10.1021/ac1006552.

35. Kind T, Liu KH, Lee DY, DeFelice B, Meissen JK, Fiehn O. LipidBlast in silico tandem mass spectrometry database for lipid identification. Nat Methods. 2013; 10: 755-8. https:// doi.org/10.1038/nmeth.2551.

36. Kessner D, Chambers M, Burke R, Agus D, Mallick P. ProteoWizard: open source software for rapid proteomics tools development. Bioinformatics. 2008; 24: 2534-6. https://doi.org/10.1093/bioinformatics/btn323.

37. Smith CA, Want EJ, O'Maille G, Abagyan R, Siuzdak G. XCMS: processing mass spectrometry data for metabolite profiling using nonlinear peak alignment, matching, and identification. Anal Chem. 2006; 78: 779-87. https://doi. org/10.1021/ac051437y. 